Reprod. Nutr. Dévelop., 1980, 20 (5 B), 1633-1639.

\title{
Comportement alimentaire comparé des ovins et des bovins. Relation avec la digestion des aliments
}

\author{
par Brigitte CARLE, J. P. DULPHY \\ avec la collaboration technique de L. L'HOTELIER, G. MOINS et A. OLLIER \\ Laboratoire des Aliments, INRA, \\ Theix, Saint-Genès-Champanelle 63110 Beaumont, France.
}

Summary. Feeding behovior of sheep and cattle as related to food digestion.

We report some preliminary results of a trial comparing the digestibility of different rations according to the type of ruminant receiving the ration. Digestibilities of maize silage were similar but not those of an excellent quality hay. The results are discussed in relation to feeding and ruminating behavior as well as the rate of passage of indigestible particles through the digestive tract.

\section{Introduction.}

La valeur alimentaire des fourrages, produit de leur concentration en éléments nutritifs par leur ingestibilité, est habituellement déterminée sur des béliers adultes castrés (INRA, 1978, p. 471) et appliquée à diverses catégories de Ruminants. Mais dans le cas d'animaux en production, ces valeurs doivent être corrigées pour tenir compte de l'apport d'aliment concentré et de leur niveau d'alimentation (INRA, 1978). En outre, il n'est pas certain que la digestibilité des fourrages soit la même suivant les différentes espèces de Ruminants alimentés à volonté, eu égard aux travaux bibliographiques récents de Playne (1978). C'est pourquoi, nous avons mis en place une importante étude, pour mesurer les différences éventuelles d'ingestibilité et de digestibilité entre diverses catégories d'ovins et de bovins, et pour essayer d'en analyser la nature. Pour ce faire, nous avons parallèlement mesuré le comportement alimentaire des ovins et des bovins à l'auge, qui a fait jusqu'ici l'objet d'un nombre réduit d'études (Dulphy, Remond et Theriez, 1979), ef la vitesse de transit des résidus indigestibles chez ces deux espèces de ruminants.

\section{Matériel et méthodes.}

Animaux. - Quatre catégories d'animaux ont été utilisées : des béliers adultes castrés pesant $65 \mathrm{~kg}$, des brebis vides taries, des taurillons de race à viande âgés de 10-12 mois et pesant $350 \mathrm{~kg}$, des vaches laitières gestantes en fin de lactation produisant en moyenne par jour $15 \mathrm{~kg}$ de lait à $4 \mathrm{p} .100$. Tous ces animaux ont été maintenus par groupe de 6 en cage ou en stalle à métabolisme. 
Fourrages. - Nous rapportons les mesures relatives aux deux premiers fourrages étudiés en nous limitant aux mesures effectuées sur les fourrages distribués seuls et à volonté (5 à 10 p. 100 de refus) : un ensilage de maïs avec urée et un foin de ray-grass (1er cycle) récolté au stade début épiaison et ventilé en grange. En complément du foin, 6 p. 100 de tourteau d'arachide ont été distribués sur la base de la matière sèche ingérée, afin que l'apport azoté total et le rapport PDIE/PDIN ne constituent pas des facteurs limitants de l'ingestibilité, ni de la digestibilité.

Mesures. - L'adaptation des animaux à leur régime a duré trois semaines, qui ont précédé les 10 jours de mesures. Les quantités de fourrage volontairement ingérées ont été mesurées, ainsi que la digestibilité de la matière sèche et des principaux constituants de ce fourrage.

L'enregistrement du comportement alimentaire des animaux a été effectué pendant 5 jours selon la technique de Ruckebusch (1963) ; la vitesse de transit de la fraction indigestible du foin de ray-grass a été appréciée par la technique des particules colorées selon la méthode de Balch (1950) et de Castle (1956) sur deux animaux de chaque catégorie.

\section{Résultats.}

Composition des fourrages. - La composition des deux aliments est présentée dans le tableau 1. Les teneurs en MAT sont plus faibles dans le cas du foin, mais restent suffisamment élevées pour ne limiter ni l'ingestibilité, ni la digestibilité du fourrage.

TABLEAU 1

Composition chimique des fourrages ingérés

\begin{tabular}{|c|c|c|}
\hline & $\begin{array}{c}\text { Ensilage de maïs } \\
(† \text { urée })\end{array}$ & $\begin{array}{c}\text { Foin de Ray-grass } \\
(+6 \text { p. } 100 \text { TA })\end{array}$ \\
\hline $\begin{array}{l}\text { Teneur en matière sèche }(p .100) \ldots \ldots \ldots \ldots \ldots \ldots \\
\text { Teneur en cendres (p. } 100 \mathrm{MS}) \ldots \ldots \ldots \ldots \ldots \ldots \\
\text { Teneur en matières azotées totales }(\mathrm{p} .100 \mathrm{MS}) \ldots \ldots \ldots \\
\text { Teneur en cellulose brute (p. } 100 \mathrm{MS}) \ldots \ldots \ldots \ldots \ldots \ldots \\
\text { Teneur en amidon }(\mathrm{p} .100 \mathrm{MS}) \ldots \ldots \ldots \ldots \ldots \ldots \ldots\end{array}$ & $\begin{array}{r}34,0 \\
5,3 \\
11,6 \\
18,4 \\
37,4\end{array}$ & $\begin{array}{r}89,4 \\
7,5 \\
10,9 \\
27,5 \\
-\end{array}$ \\
\hline
\end{tabular}

Quantités ingérées. - Les quantités de MS ingérées, rapportées au poids métabolique, sont en moyenne de 56,7 pour les ovins contre 76,8 pour les taurillons et 111,0 pour les vaches laitières. Les écarts sont donc très importants. Entre ovins, les quantités de MS ingérées ont néanmoins beaucoup varié suivant le fourrage (tabl. 2 et 3 ).

Activités alimentaires. - Les durées journalières d'ingestion ef de rumination n'ont pas été différentes entre les deux catégories d'ovins pour les deux fourrages étudiés (tabl. 2 et 3 ). Ils ont simplement passé un peu plus de temps à ingérer et à ruminer le foin que l'ensilage de maïs (en moyenne respectivement 271 et 545 min 
TABLEAU 2

Quantités de Matières sèches ingérées ef comporfement alimentaire ef mérycique des animaux

Taurillons

Vaches laitières

Quantités de MS ingérées $\left(g / k g p^{0,75}\right) \ldots \ldots \ldots \ldots$. Durée journalière d'ingestion (min/jour) ........ Durée unitaire d'ingestion $\left(\mathrm{min} / \mathrm{g} / \mathrm{kg} \mathrm{P}^{0,75}\right) \ldots \ldots \ldots$ Durée journalière de rumination ( $\mathrm{min} /$ jour) ... Durée unitaire de rumination $\left(\mathrm{min} / \mathrm{g} / \mathrm{kg} \mathrm{P}^{0,75}\right.$ ) ...

Durée journalière de mastication ( $\mathrm{min} /$ jour) .....

Durée unitaire de mastication ( $\mathrm{min} / \mathrm{g} / \mathrm{kg} \mathrm{P}^{0,75}$ ) ...

\begin{tabular}{|c|c|c|c|}
\hline $48,3 \pm 3,0$ & $63,6 \pm 7,7$ & $76,9 \pm 4,3$ & $111,3 \pm 12,3$ \\
\hline $\pm 35 a$ & $\pm 14^{a}$ & $\pm 61 b$ & $\pm 43^{\circ}$ \\
\hline $4,89 \pm 0,46 a$ & $3,40 \pm 0,65^{b}$ & $4,80 \pm 0,91 a$ & $3,07 \pm 0,29 b$ \\
\hline $\pm 68 a$ & $\pm 48 a$ & $\pm 43 b$ & $\pm 69 a b$ \\
\hline $10,56 \pm 1,56 a$ & $8,19 \pm 1,06 b$ & $5,19 \pm 0,65 c$ & $3,90 \pm 0,54 d$ \\
\hline \pm 64 & \pm 54 & \pm 54 & \pm 89 \\
\hline $5 \pm$ & 1,6 & $9,99 \pm 1,05^{b}$ & $0,56 c$ \\
\hline
\end{tabular}

** $P<0,01 \quad$ *** $P<0,001$.

$a, b, c, d$ : les valeurs affectées de la même lettre ne sont pas significativement différentes.

TABLEAU 3

Quantités de Matières sèches ingérées et comportement alimentaire ef mérycique des animoux (suite)

Foin de ray-grass

( + Tourteau d'arachide)
Béliers
Brebis
Taurilions
Vaches laitières Test

Quantités de MS ingérées $\left(g / \mathrm{kg} \mathrm{P}^{0,75}\right) \ldots \ldots \ldots \ldots$ Durée journalière d'ingestion (min/jour) ..... Durée unitaire d'ingestion $\left(\mathrm{min} / \mathrm{g} / \mathrm{kg} \mathrm{P}^{0,75}\right) \ldots \ldots$.

Durée journalière de rumination (min/jour) ...

Durée unitaire de rumination ( $\left.\mathrm{min} / \mathrm{g} / \mathrm{kg} \mathrm{P}^{0,75}\right)$. Durée journalière de masfication ( $\mathrm{min} /$ jour) .... Durée unitaire de mastication ( $\mathrm{min} / \mathrm{g} / \mathrm{kg} \mathrm{P0,75}$ ).

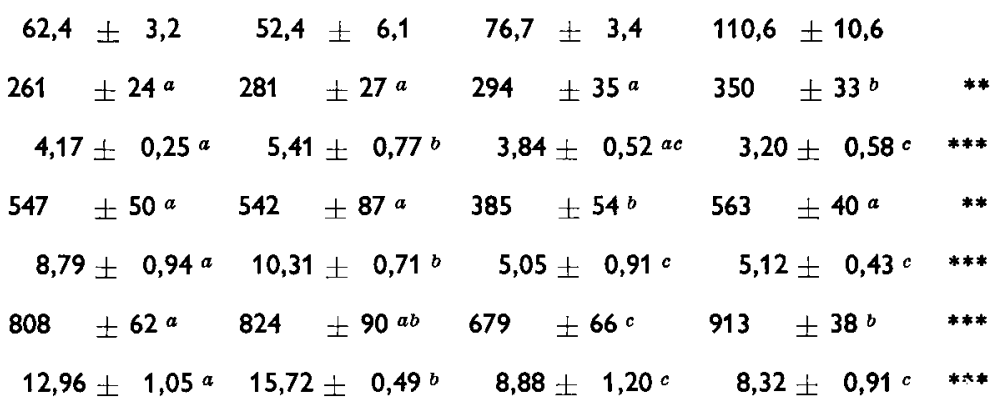

**P $\mathrm{P}<0,01 \quad * * * \mathrm{P}<0,001$.

$a, b, c, d$ : les valeurs affectées de la même lettre ne sont pas significativement différentes.

contre 225 et $512 \mathrm{~min}$ ) mais les différences sont seulement significatives au niveau de la durée d'ingestion chez les brebis.

Les durées d'ingestion des bovins ont été significativement supérieures à celles des ovins dans le cas de l'ensilage de maïs; ceci n'est pas vérifié pour les taurillons dans le cas du foin de ray-grass (tabl. 2 et 3). 
Les durées de rumination ont été, en moyenne, plus faibles pour les bovins que les ovins (444 contre $530 \mathrm{~min}$ ) ; mais les différences entre les vaches et les ovins ne sont pas significatives pour les deux fourrages ; par contre, les taurillons ont passé beaucoup moins de temps à ruminer le foin de ray-grass $(P<0,01)$ que ne l'ont fait les autres catégories d'animaux (385 contre $547 ; 542$ et $563 \mathrm{~min}$ ).

En fin de comple, les durées journalières de mastication sont comparables entre les ovins et les bovins pour l'ensilage de maïs, mais dans le cas du foin significativement plus élevées chez les vaches et inférieures chez les taurillons (tabl. 2 et 3 ).

Les durées unitaires d'ingestion, de rumination ef de mastication ont varié significativement entre les différentes catégories de ruminants suivant le fourrage, sans qu'il soit permis de regrouper d'une part les valeurs obtenues sur les deux catégories d'ovins, et d'autre part celles pour les bovins (tabl. 2 et 3). Par contre, ces durées unitaires ont varié de façon inverse avec les quantités ingérées par les animaux (fig. 1).

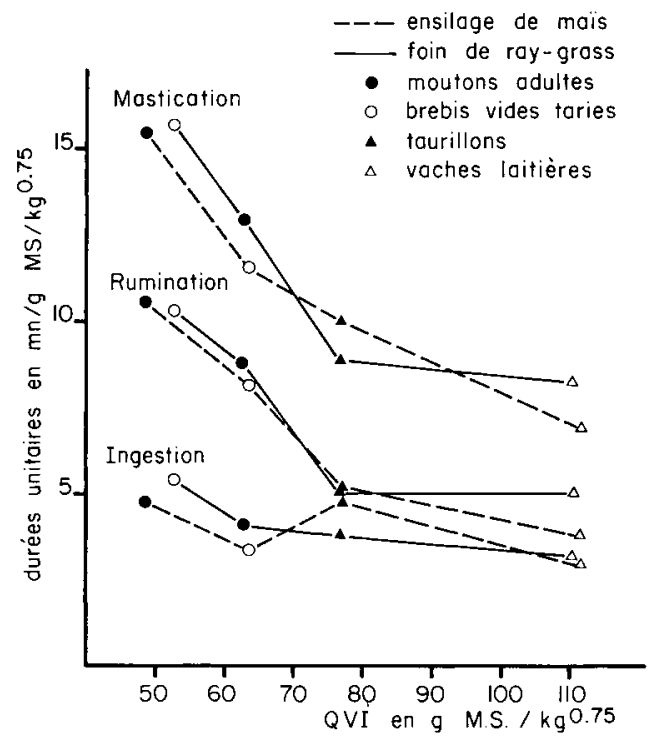

FIG. 1. - Relation entre les durées unitaires d'ingestion, de rumination, de mastication (en $\mathrm{min} / \mathrm{g} \mathrm{MS} / \mathrm{kg}^{0,75}$ ) et les quantités volontairement ingérées (QVI en g MS/kg $\mathrm{P}^{0,75}$ ).

\section{Ceci explique :}

- que les durées unitaires soient plus élevées chez les moutons par rapport aux brebis pour l'ensilage de maïs $(P<0,01)$ et inversement pour le foin de ray-grass $(P<0,01)$;

- que les temps consacrés à l'ingestion, la rumination et surtout la mastication pour une quantité donnée de fourrage soient inférieurs chez les bovins par rapport aux ovins, et foujours de façon significative chez les vaches.

Néanmoins, on ne peut vérifier à partir de ce nombre restreint de résultats, que la relation entre les durées unitaires et les quantités ingérées soit la même pour les deux espèces de ruminants.

Les durées unitaires d'ingestion, de rumination et de mastication ont été infé- 
rieures pour le foin par rapport d̀ l'ensilage de maīs $(P<0,05)$ chez les moutons : elles sont au contraire supérieures dans ce cas $(P<0,01)$ chez les brebis et chez les vaches pour les durées de rumination $(P<0,01)$ et de mastication $(P<0,05)$.

Les résultats enregistrés sur taurillons sont quelque peu surprenants, au niveau de l'ingestion de l'ensilage de maïs et au niveau de la rumination et de la mastication du foin de ray-grass (fig. 1), mais les différences entre les deux fourrages ne sont pas significatives, quel que soit le paramètre considéré.

Digestibilité (tabl. 4). - La digestibilité de la matière organique de l'ensilage de maïs n'a pas été significativement différente suivant les catégories animales, malgré un écart de 3,3 points en faveur des brebis par rapport aux taurillons. Cependant, la digestibilité de l'amidon (mesurée par lol) a été bien plus élevée chez les ovins que

TABLEAU 4

Digestibilité des fourrages par les différentes catégories d'animaux

\begin{tabular}{|c|c|c|c|c|c|}
\hline & Béliers & Brebis & Taurillons & $\begin{array}{l}\text { Vaches } \\
\text { laitières }\end{array}$ & Test $F$ \\
\hline \multicolumn{6}{|l|}{ Ensilage de maïs } \\
\hline $\begin{array}{l}\text { Digestibilité }(p .100) \\
\text { Matière organique } \ldots \ldots \ldots \\
\text { Amidon } \ldots \ldots \ldots \ldots \ldots \\
\text { Cellulose brute } \ldots \ldots \ldots \ldots\end{array}$ & $\begin{array}{c}71,8 \pm 0,9 \\
99,0 \\
62,0 \pm 1,6 a\end{array}$ & $\begin{array}{l}73,3 \pm 1,6 \\
98,2 \\
64,5 \pm 2,9 a b\end{array}$ & $\begin{array}{c}70,0 \pm 3,0 \\
91,2 \\
64,1 \pm 3,6 a b\end{array}$ & $\begin{array}{l}71,6 \pm 0,9 \\
86,4 \\
66,9 \pm 1,2 b\end{array}$ & $\begin{array}{c}\text { NS } \\
(1) \\
*\end{array}$ \\
\hline \multicolumn{6}{|l|}{ Foin } \\
\hline $\begin{array}{l}\text { Digestibilité (p. 100) } \\
\text { Matière organique ....... } \\
\text { Cellulose brute ........ } \\
\text { Temps de séjour moyen de } \\
\text { l'indigestible }(h) \ldots \ldots \ldots \ldots\end{array}$ & $\begin{array}{c}67,9 \pm 1,6 a \\
66,1 \pm 2,4 a \\
53\end{array}$ & $\begin{array}{c}71,1 \pm 2,5 b \\
67,4 \pm 4,3 a \\
54\end{array}$ & $\begin{array}{c}73,7 \pm 1,7 b c \\
74,5 \pm 2,2 a \\
71\end{array}$ & $\begin{array}{c}74,6 \pm 1,2 c \\
72,6 \pm 1,6 b \\
75\end{array}$ & $\begin{array}{l}* * * \\
* * *\end{array}$ \\
\hline
\end{tabular}

$* \mathrm{P}<0,05 \quad * * * \mathrm{P}<0,001$.

$a, b, c, d$ : les valeurs affectées de la même lettre ne sont pas significativement différentes.

$\left({ }^{1}\right)$ Analyse par lot.

chez les bovins (en moyenne 98,6 contre 88,8 ). Ceci est confirmé par la présence de grains ef de fragments de grains de maïs dans les fèces de bovins : 6,4 p. 100 ef 10,0 p. 100 des grains ingérés pour les taurillons ef les vaches. A l'inverse, la digestibilité du résidu obtenu en retranchant à la matière organique les matières azotées totales, l'amidon et la cellulose brute, a été bien supérieure pour les bovins ( 58,2 contre 43,2 p. 100).

La digestibilité de la matière organique du foin a été plus élevée chez les bovins (en moyenne 74,1) que chez les béliers $(67,9)(P<0,01)$, et parmi les ovins, la digestibilité supérieure $(P<0,05)$ chez les brebis est vraisemblablement à relier aux quantités ingérées plus faibles pour ces animaux. Les différences entre espèces animales sont nettes au niveau de la digestibilité de la cellulose brute, supérieure chez les bovins.

Temps de séjour des particules indigestibles. - Le temps de séjour moyen des particules colorées dans le tube digestif des animaux recevant le foin a été très différent (tabl. 4) entre les ovins et les bovins ( $53 \mathrm{~h} 30$ contre $73 \mathrm{~h}$ ). 


\section{Discussion.}

Les différences importantes de quantités ingérées exprimées par rapport au poids métabolique selon le type d'animal sont conformes aux résultats du groupe de travail du Département d'Elevage des Ruminants (1979). S'il est intéressant de constater que, malgré ces différences, les durées journalières de mastication sont identiques entre les ovins et les bovins recevant de l'ensilage de maïs, dans le cas d'un foin de ray-grass, le temps consacré à la mastication varie non seulement d'une espèce à l'autre, mais particulièrement entre les deux types de bovins. Ces écarts, ainsi que les différences de durées d'ingestion ef de rumination entre les catégories animales suivant la nature du fourrage, devront être précisés par l'étude d'autres fourrages.

Pour mastiquer une quantité donnée de foin, les bovins mettent beaucoup moins de temps que les ovins. On peut alors admettre que la réduction du fourrage en fines particules est moins poussée chez les bovins ; ceci est d'ailleurs confirmé par le fait que la faille des particules de fourrage passant dans le feuillef est plus élevée pour les bovins que pour les ovins (Grenet, 1966, 1970 ; Thomas et Campling, 1977 ; Michalet-Doreau, non publié).

La digestibilité de la cellulose brute supérieure chez les bovins résulte donc très vraisemblablement du temps de séjour plus long de cette cellulose dans leur tractus digestif. Les résultats de Thomas et Campling (1977) obtenus sur des animaux recevant du foin de ray-grass à volonté ne confirment pas nos observations ; les différences entre moutons ef vaches au niveau de la digestibilité de la matière organique $(60,4$ et $61,4)$, de celle de la cellulose bruie $(69,0$ et 69,7$)$ et du temps de séjour moyen $(60$ et 67 h) ne sont pas significatives.

Dans le cas de l'ensilage de maïs, la digestibilité incomplète de l'amidon par les bovins pénalise l'utilisation digestive par ces animaux de la matière organique de ce fourrage, qui n'est pas significativement différente entre espèces. Ces différences de digestibilité de l'amidon peuvent s'expliquer par les différences de durées unitaires de mastication, et dépendent notamment de l'importance de la rumination, comme l'ont montré Wilson, Adeeb et Campling (1973) ; le pourcentage de grains excrétés par rapport aux grains ingérés a d'ailleurs varié entre les taurillons ef les vaches, inversement aux durées unitaires de mastication ef aux digestibilités de l'amidon mesurées pour ces types de bovins.

\section{Conclusion.}

Il existe donc des différences importantes au niveau de la digestibilité des constituants des deux fourrages étudiés, entre l'espèce ovine ef l'espèce bovine. La capacité des ovins à mastiquer plus longtemps une quantité de fourrage donnée favorise vraisemblablement une vitesse de digestion dans le rumen plus élevée que chez les bovins (Meissner ef al., 1979) ; pour ces derniers, le temps de séjour plus long des aliments dans le fube digestif, et notamment dans le réticulo-rumen, permet de compenser ce « handicap » au niveau de la digestion des membranes. Il serait important de pouvoir préciser ces observations, par la mesure d'un autre facteur de l'intensité de la digestion des fourrages, qui est l'activité cellulolytique du rumen. 
D'autre part, il existe aussi des différences importantes entre les catégories animales d'une même espèce, et par exemple au niveau de la capacité des taurillons et des vaches à digérer les grains de l'ensilage de maïs.

Nous poursuivrons donc ce travail, en comparant l'efficacité digestive ef les facteurs de la digestion pour différentes catégories de ruminants pour une gamme de fourrages plus étendue et plus variée, ce qui devrait nous permettre d'estimer la digestibilité des aliments par les ruminants en production à l'aide des mesures habituellement effectuées sur des béliers adultes castrés.

$$
\begin{aligned}
& \text { Journées Ingestion-Digestion-Absorption } \\
& \text { de l'Association française de Nutrition, } \\
& \text { Paris, 15-16 novembre } 1979 .
\end{aligned}
$$

\section{Références}

BALCH C. C., 1950. Factors affecting the utilization of food by dairy cows. I. - The rate of passage of food through the digestive tract. Bri. J. Nutr., 4, 361-388.

CASTLE E. J., 1956. The rate of passage of foodstuffs through the alimentary tract of the goat. I. - Studies on adult animals fed on hay and concentrates. Bri. J. Nutr., 10, 15-23.

DÉPARTEMENT D'ÉLEVAGE DES RUMINANTS, 1979. Le système des unités d'encombrement pour les bovins. Bull. Tech. CRZV Theix, INRA, no 38, décembre 1979.

DULPHY J. P., REMOND B., THERIEZ M., 1979. Ingestive behaviour and related activities in ruminants. Ve Symp. Ruminant physiology, Clermont-Ferrand, sept. 1979.

GRENET E., 1966. Les particules végétales des fèces de moutons. Ann. Zootech., 15, 303-312.

GRENET E., 1970. Taille et structure des particules végétales au niveau du feuilleł et des fèces chez les bovins. Ann. Biol. anim. Bioch. Biophys., 10, 643-657.

I.N.R.A., 1978. Alimentation des ruminanis. Ed. INRA Publ., Route de St-Cyr, 78000 Versailles.

MEISSNER H. H., PIENAAR J. P., LIEBENBERG L. H. P., ROUX C. Z., 1979. Rumen flow dynamics and voluntary infake in ruminants. Ann. Rech. vet., 10, 219-222.

PLAYNE M. J., 1978. Estimation of the digestibility of low-quality hays by cattle from measurements made with sheep. Anim. Feed Sci. Technol., 3, 51-55.

RUCKEBUSCH Y., 1963. Recherches sur la régulation centrale du comportement alimentaire chez les ruminants. Th. Doct. Sci., Université de Lyon.

THOMAS S., CAMPLING R. C., 1977. Comparisons of some factors affecting digestibility in sheep and cows. J. Brit. Grassl. Soc., 32, 33-41.

WILSON G. F., ADEEB N. N., CAMPLING R. C., 1973. The apparent digestibility of maize grain when given in various physical forms to adult sheep and cattle. J. agric. Sci., Camb., 80, 259-267. 